\title{
Easy Diagnosis of Myeloblastic Leukemia by Combined Use of Sat $=$ =Sekiy a's Original Method of Copper Peroxidase Reaction and the Tohoku Pediatric Method, its Modification. The Characteristic Feature of Myeloblasts First Demonstrated by Lambin and Leto in Sato=Sekiy a's Stain.*
}

36th Hematological Paper.

By

Shizuo Kimura.

(木村静堆)

(From the Department of Pediatrics, Faculty of Medicine, Tohoku Imperial University, Sendai.

Director: Prof. A. Sato.)

Myeloblastic leukemia is very often diagnosed as lymphatic leukemia, especially by those who do not utilize either oxidase or peroxidase reaction. Even for those who make use of an oxidase or a peroxidase method the diagnosis is difficult in some cases of myeloblastic leukemia, especially in aleukemic myelosis. I report here of a very easy method (easy especially for beginners in hematology) of diagnosing a case of myeloblastic leukemia. The present paper treats of the method.

\section{Method Used.}

A fresh blood film is stained by Sato and Sekiya's original copper peroxidase reaction ${ }^{1)}$, and another by the Tohoku Pediatric Method ${ }^{2)}$, itself a modification of the original. As to the technique see the foot-notes (p. 353-354).

* Read at the 23rd General Meeting of the Tohoku-Medical Society, Sendai, October, 1938.

1) A. Sato and S. Sekiy a, Tohoku Igaku-Zassi, 1923, 6, 534 ; Tohokn J. Exp. Med., 1926, 7, 111 ; Tohoku J. Exp. Med., 1936, 29, 92.

A. The original method, published in 1923 (p. 359). 
The picture in Sato-Sekiya's peroxidase method is different from that in the Tohokn Pediatric Method, but the difference is not essential. We are wont to use the latter method ${ }^{21}$, a modification of the former, for daily routine work.

Reigents used :

(1) Solution A. $0.5 \%$ copper sulphate solution.

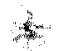

(2) Solution B. Rub 0.2 grms. of benzidine with a few drops of water in a mortar. Add 200 c.c. of water at room temperature and filter. To the filtrate add 4 drops of $3 \%$ hydrogen peroxide. If kept in a brown bottle in the dark when not in use, this preparation will last a year, although precipitation will begin to occur soon after preparation.

(3) Solution C (Counter stain). Either 1\% safranin, 1\% neutral red, or carbol fuchsin diluted $1: 5$. Safranin is by far the best of these connter stains.

Nethod used:

To a fresh, dry blood smear apply Solution A. . Pour off most of it and apply solution. $\mathrm{B}$ for two minutes. Then wash thoroughly with water, and for counter staining add Solution $\mathrm{C}$ at once and let stand for two minutes before washing.

Result:

The peroxidase granules of the lencocytes are stained a deep bluish green. The lymphocytes and the lymphoid cells show no trace of blue or green, but appear red because of their red-staining nuclei.

B. The original method in its present technique.

The preparation of Solution B is simplified: Rub 0.2 grms. of benzidine with a few drops of water in a mortar. Add 200 c.c. of water at room temperature and shake vigorously. Without filtering it, add 4 drops of $3 \%$ hydrogen peroxide to the benzidine suspension. Keep in a brown bottle.

Technique :

To an air-dried blood film apply Solution A. Pour off most of it at once and apply Solution B for two minutes. Then Solution C (1\% safranin) is applied for 4-5 minutes. Wash thoroughly with water.

2) A. S ato, T. Suzuki and Ry. Shibata, Tohoku J. Exp. Med., 1934, 24, 195; Tohoku J. Exp. Med., 1936, 29, 92.

The Tohoku Pediatric Method.

Reagents used:

Solution A. Copper sulphate solution, 10 per cent.

Solution B. Saturated solution of benzidine, to which $3 \%$ hydrogen peroxide was added in the amount of 2 drops per 100 c.e.

Solution C. Safranin, aqueous solution, 1 per cent.

Technique:

Pour Solution A half full on a tresh, air-dried blood smear, and then a few drops of Solution $C$ and mix. After a lapse of four or five minutes pour off most of them and apply Solution $B$ on the smear and wait about one minute. Wash thoroughly with running water and then dry.

Purpose :

This modification is mostly used in daily practice in our Laboratory for the following different purposes :-

a. For the variety differentiation of leucocytes [Cf. 50th ${ }^{2}$ ) and $63 \mathrm{rd}^{3}$ ) Reports ot the Peroxidase Reaction].

b. For the nuclear shift. Paper].

e. For the nuclear topical diagnosis (Cf. the $2 \mathrm{nd}^{\mathrm{s}}$ ) and $16 \mathrm{th}^{5}$ ) Hematological

3) A. Sato, T. Suz $\mathbf{k} i$ and Ry. Sh ibata, Tohoku J. Exp. Med., 1936, 28, 285.

4) T. Suzuki, Tohok u J. Exp. Med, 1936, 29, 108.

5) M. Shind o, Y. Ko kuboand Sh. Kimura, TohokuJ. Exp. Med., 1988, 33, 304. 
In the present special case both methods-the original and its modification -are used. The Giemsa stain is not necessary.

\section{Result.}

Compare the film stained by the original method with the other stained by the Tohoku Pediatric Method. If one sees myeloblasts or such like cells (Fig. 1, a) -in the film stained by the latter, and sees in the film by Sato-Sekiya's original method such like cells with "Peroxidase-positive nucleus" (Fig.2,a)-, then the case is highly probably myeloblastic leukemia. By the expression "peroxidase-positive nucleus" we do not mean that the nucleus of myeloblasts are peroxidasepositive. The peroxidase-positive part corresponds to the central part of the nucleus, but not entirely. The peroxidase-positive part is about 2 or 3 sizes smaller than the nucleus. But for convenience sake we speak of "peroxidase-positive nucleus." Peroxidase-positive "centrosphere" may be a better expression.

\section{Mycloblasts with "Peroxidase-positive nucleus."}

Myeloblasts with peroxidase-positive centrosphere were described as early as 1930 by La m bin and Le to ${ }^{6}$, who applied Sato-Sekiya's original copper peroxidase method to a case of acute leukemia and found it to be a case of myeloblastic leukemia. They then described the characteristic peroxidase features of myeloblasts as in the Footnote.*

The peroxidase granules on the nucleus do not cover the full scope of that nucleus; the peroxidase-positive part is, as described above, smaller than the nucleus itself-so to speak-two or three sizes smaller (Cf. Fig. 2). The peroxidase granules in the central part of nucleus are delicate and abundant, and those on one side or corner of the cell body are coarse and rather scarce. The number of granules of the central part are larger than was reported by La m bin and Leto (Cf. Footnote $\left.{ }^{*}\right)$.

6) P. L a m bin and A. L e to, Rev. belge d. Sc. Méd., 1930, 2, 236.

*Nous avons constaté que le nombre des granulations peroxydasiques n'était pas aussi grand dans les myéloblastes que dans les éléments granuleux. Alors que ceuxci présentaient une quantité considerable de granulations presque juxtaposées, réparties dans tout le protoplasme, environ les denx tiers des myéloblastes ne présentaient qu'un petit nombre de granules (de 15 à 25 le plus souvent) groupés dans le voisinage de la centrosphére, quelques rares grains étant disséminés dans le reste du cytoplasme. 


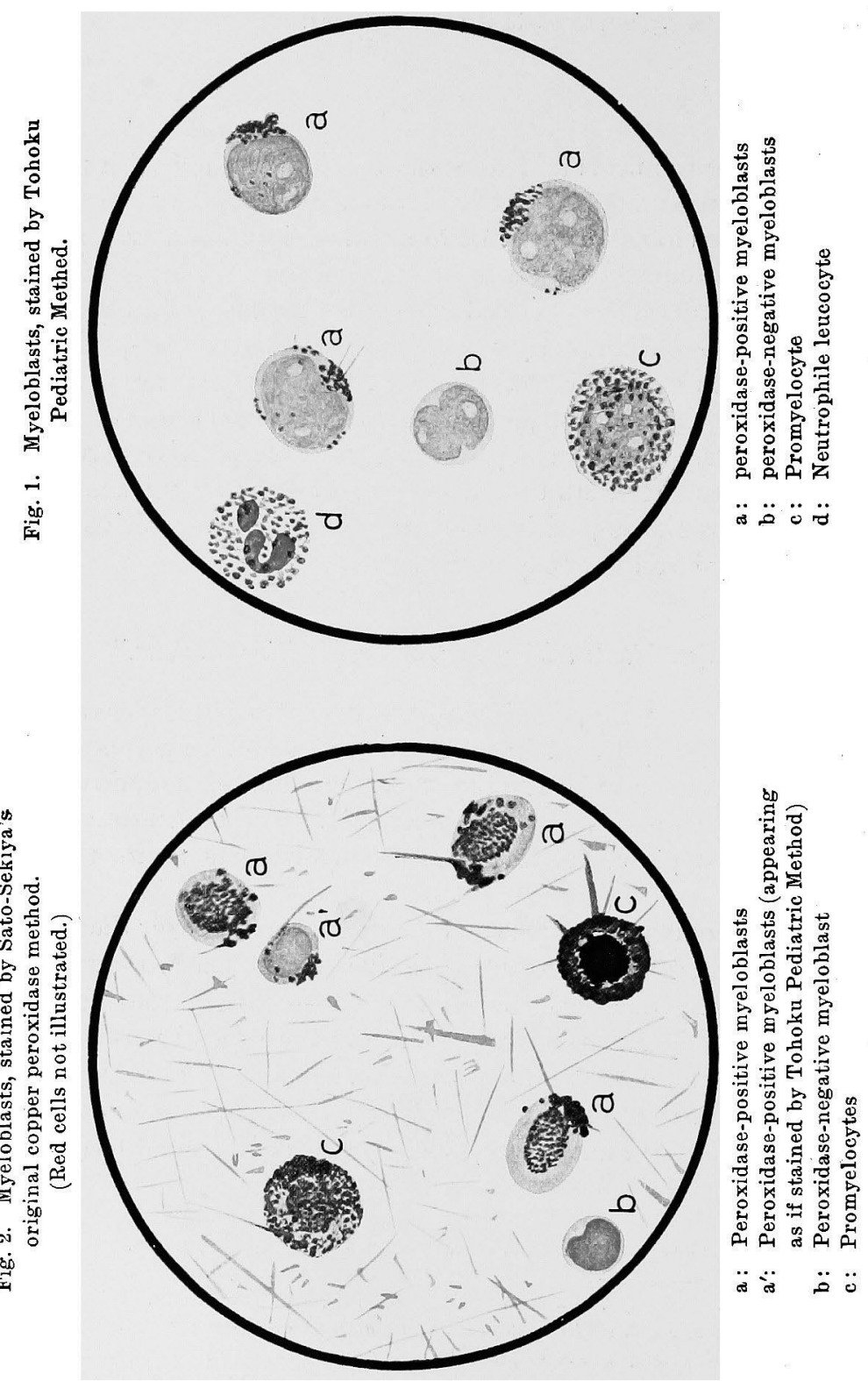


Usually the difference between both methods of peroxidase stain -Sato-Sekiya's original method ${ }^{1 /}$ and Tohoku Pediatric Method ${ }^{2}$ - may be said to be that of coarseness or the density of peroxidase granules, so that for daily routine hematological work, the Tohoku Pediatric Method is preferred, because less dense and smaller peroxidase granules allow us to recognize the outline and features of nucleus much more distinctly than with Sato-Sekiya's original method.

But in myeloblastic leukemia the difference is essential. And the difference is due to the different concentration of copper sulphate solution used in Sato-Seki ya's original method and the Tohoku Pediatric Method. The copper sulphate solution is $0.5 \%$ in the former and $10 \%$ in the latter. It became thus important to try copper sulphate solutions of different concentration. I stained the blood films of myeloblastic leukemia with the technique of Sato and Seki ya's peroxidase reaction, using $10 \%$ copper sulphate solution instead of $0.5 \%$ solution, and obtained almost the same picture as shown by the Tohoku Pediatric Method, but the peroxidase reaction was stronger and the granules were larger than in the use of the Tohoku Pediatric Method. With the use of $0.5 \%$ copper sulphate solution instead of $10 \%$ solution, the technique of the Tohoku Pediatric Method gave the same picture of myeloblasts as in the use of the original method. This result is enough to show that the difference of the picture of peroxidase stain of myeloblasts between Sato-Sekiy a's original method and the Tohoku Pediatric Method is almost exclusively due to the different concentration of copper sulphate solution, the other reagents being of no important account. So I tried to stain the blood films of myeloblastic leukemia with Sato-Sekiya's original method, only changing the concentration of the copper sulphate solution. The result is shown in the Table (Cf. Table).

As will be observed in the Table, myeloblasts with "peroxidasepositive nucleus" were seen in $6 \%$ of all myeloblasts in the case when $10 \%$ copper sulphate solution was used, in $22 \%$ when $5 \%$ solution was used, in $31 \%$ when $2.5 \%$ solution was used, in $54 \%$ when $1 \%$ solution was used, and in $61 \%$ when $0.5 \%$ copper sulphate solution was used as in the case of the original Sato and Sekiy a method, in $47 \%$ when $0.25 \%$ solution was used, in $20 \%$ when $0.1 \%$ solution was used, in $12 \%$ when $0.05 \%$ solution was used, in $8 \%$ when $0.025 \%$ solution was used, and in only $3 \%$ when $0.01 \%$ solution was used.

The size of peroxidase granules was always more coarse and less numerous when solutions of lower concentrations were used, especially 


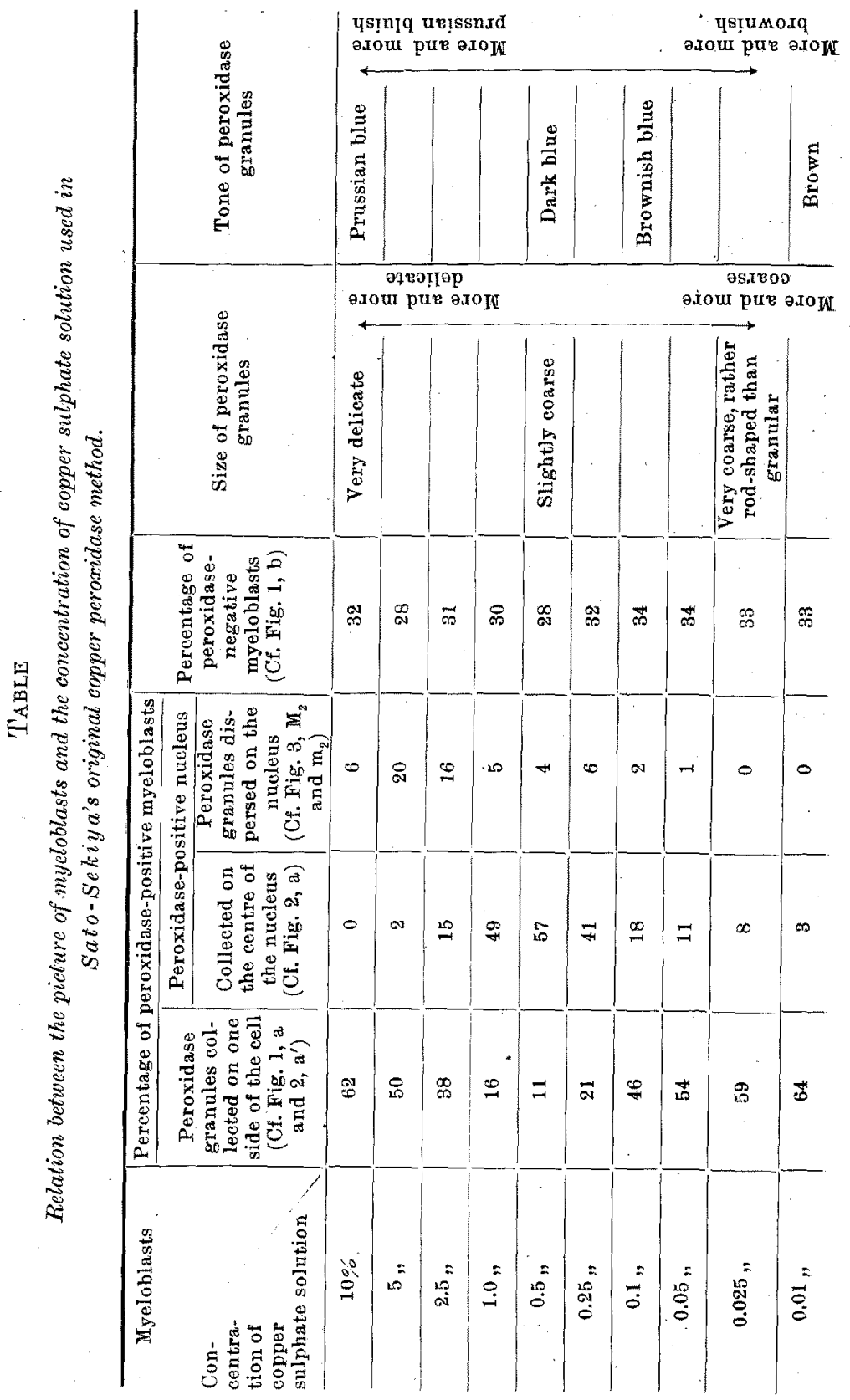


when the percentage was below $0.05 \%$. In these cases the peroxidase granules were only very few and rod-shaped rather than granular, and the colour of the peroxidase granules ceased to become blue, showing a tendency to dark brown colour.

The two methods offer different pictures of myeloblasts, as described above, though one cannot by any means say that all the myeloblasts will present the essential difference. Sato, Suzuki and Shibata ${ }^{3)}$ of our Laboratory described the peroxidase picture of myeloblasts in the use of Tohoku Pediatric Method and stated that most peroxidase granules appear to have been drawn in the majority of instances to one side or corner of the cell body (sometimes to both opposite sides) and remain collected there. I always see the same picture by that method. They further stated in the same paper that the appearance of myeloblasts under the original Sato-Sekiya's peroxidase stain is similar to that under the Tohoku Pediatric Method. Such statement of theirs seems to be at variance with what I have described in the present paper. But we must take into consideration the following fact :-

An experienced hematologist will recognize different kinds of one and the same blood disease of myeloblastic leukemia, and it is not difficult to suppose that there are cases of myeloblastic leukemia, sometimes having myeloblasts with "peroxidase-positive nucleus" and at other times with no such "nucleus", be the concentration of copper sulphate solution $10 \%$ or $0.5 \%$. Even in one and the same case we see both kinds of myeloblasts (Cf. Fig. 2, a and a'), though the methods are different.

\section{Appendix.}

We have seen that myeloblasts show "peroxidase-positive nucleus" with the original Sato-Sekiya's copper peroxidase method. But promyelocytes can show such a feature under this method, while the Tohoku Pediatric Method fails to show it (Cf. Fig. 1, b and Fig. 2, b). Myelocytes and metamyelocytes will not show such a picture even under the original method. In other words, these two kinds of cells show almost the same peroxidase picture under both methods.

\section{Conclusions.}

Myeloblastic leukemia can be diagnosed very easily even by a begirner in hematology by the combined use of the Sato-Sekiya's ori- 
ginal copper peroxidase method and the Tohoku Pediatric Method, because the pictures of myeloblasts are essentially different under both methods.

If we see in one film many cells with such pictures as in Fig. $3, M_{1}$ by the Tohoku Pediatric Method, and in another film many cells with such pictures as in Fig. 3, $m_{1}$ with Sato-Sekiya's original copper peroxidase nethod, highly probably it is one of myeloblastic leukemia.
Fig. 3. Diagram of peroxidase granules of myeloblasts and promyelocytes (the outline of sucleus is not drawn). Stained

$$
\begin{array}{cc}
\text { Tohoku } & \text { Sato- } \\
\text { Pediatric } & \text { Sekiya's } \\
\text { Methiginal } \\
\text { method }
\end{array}
$$
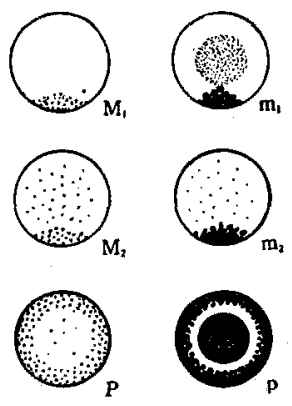

$\mathrm{M}_{1}, \mathrm{M}_{2}, \mathrm{~m}_{1}, \mathrm{~m}_{2}$ : myeloblasts P, p: promyelocytes 